\title{
Belphégor
}

\section{La Tribune de l'Histoire : la mise en récit de l'histoire dans une émission de radio populaire}

\section{Céline Loriou}

\section{(2) OpenEdition}

1 Journals

\section{Édition électronique}

URL : https://journals.openedition.org/belphegor/3725

DOI : 10.4000/belphegor.3725

ISSN : 1499-7185

Éditeur

LPCM

\section{Référence électronique}

Céline Loriou, "La Tribune de l'Histoire : la mise en récit de l'histoire

dans une émission de radio populaire », Belphégor [En ligne], 19-1 | 2021, mis en ligne le 28 juin 2021, consulté le 06 juillet 2021. URL : http://journals.openedition.org/belphegor/3725 ; DOI : https:// doi.org/10.4000/belphegor.3725

Ce document a été généré automatiquement le 6 juillet 2021.

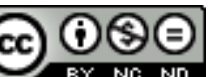

Belphégor est mis à disposition selon les termes de la Licence Creative Commons Attribution - Pas d'Utilisation Commerciale - Pas de Modification 4.0 International. 


\title{
La Tribune de l'Histoire : la mise en récit de l'histoire dans une émission de radio populaire
}

\author{
Céline Loriou
}

1 «Comment, cette passion, la communiquer au plus grand nombre? Comment faire connaître que l'histoire est un bonheur? La réponse se formula d'elle-même : il y avait la radio $»^{1}$. Si Alain Decaux, André Castelot et Jean-Claude Colin-Simard se tournent vers la radio pour créer une émission d'histoire en octobre 1951, c'est sans doute moins par amour pour ce média sonore et les possibilités qu'il réserve en matière de mise en scène de l'histoire que pour les promesses de larges auditoires qu'il offre. En effet, à cette époque, la radio connaît son âge d'or après avoir joué un rôle de premier plan pendant la guerre comme moyen d'information, de propagande mais aussi de résistance, tandis que la télévision n'en est encore qu'à ses prémices en France.

2 La Tribune de l'histoire est initialement conçue comme un magazine sur l'actualité de l'histoire, dans lequel sont passées en revue les expositions, parutions, sorties de films et dernières découvertes historiques sur le Programme parisien². Mais l'émission est surtout restée célèbre pour ses évocations scénarisées, qui remplacent définitivement le magazine à partir de 1956: des comédiens et comédiennes y rejouent les grands épisodes historiques grâce à des séquences dialoguées, entrecoupées par le récit d'un narrateur ou par les commentaires de l'un des producteurs. De temps à autre, l'évocation est précédée par une courte revue des parutions dans le domaine historique, et, à partir de la fin des années 1960, un débat de dix à quinze minutes est ajouté en fin d'émission pour mettre en perspective le sujet du jour, parfois en présence d'un ou d'une spécialiste du thème traité.

3 Des années 1950 jusqu'à l'arrêt de l'émission en 1997, la Tribune de l'histoire a traversé un demi-siècle au cours duquel la place de la radio en France a changé, avec la concurrence de la télévision, l'apparition de nouvelles chaînes, la libération des ondes 
et l'évolution des pratiques d'écoute. La discipline historique a elle aussi évolué, sous l'influence de l'école des Annales, renouvelant ses méthodes et multipliant ses champs et objets d'études. Enfin, de nouveaux lieux de vulgarisation de l'histoire ont émergé télévision, bandes dessinées, collections éditoriales spécifiques, festivals ou encore lieux de reconstitution. Malgré cela, la formule de la Tribune change peu sur l'ensemble de la période. Il est dès lors intéressant de voir comment les producteurs de l'émission ont cherché à s'approprier le support radiophonique pour rendre l'histoire accessible " au plus grand nombre». Cela suppose d'abord de s'interroger sur le type d'histoire qu'ils s'attachent à évoquer ${ }^{3}$ avant d'analyser la forme de l'émission au regard des contraintes et libertés que suggère ou impose le média radiophonique ${ }^{4}$. Bien que les producteurs assurent ne rien sacrifier à la vérité historique, les évocations reposent largement sur l'invention de dialogues entre des personnages du passé, ce qui, couplé avec le type d'histoire qu'ils mettent en avant, place La Tribune de l'histoire au cœur des désaccords entre approches universitaires et approches médiatiques de l'histoire.

\section{Le choix d'une histoire événementielle et personnifiée, contemporaine et française}

4 Pour évoquer le tout premier livre qu'il a rédigé à l'âge de 9 ans, au milieu des années 1930, Alain Decaux se souvient surtout de son titre, Le Secret du roi : «Je ne sais plus ce que pouvait recouvrir ce titre, de quel secret ni de quel roi il s'agissait. Mais c'était un titre. Et c'était un livre. De huit pages, mais un livre. $»^{5}$ Plusieurs décennies après cette première expérience d'écriture, l'attention accordée aux intitulés des évocations scénarisées de la Tribune de l'histoire est toujours là : « La vraie Lola Montès », « François $I^{\text {er }}$ et les femmes : les maîtresses ennemies ", «La marche à l'échafaud de Louis XVI ", « Pour ou contre Ponce Pilate?", "L'armistice de 1918 vu par les Allemands ». Ces titres doivent servir à piquer la curiosité des auditeurs et auditrices qui, dans les années 1950 ou 1960, ont régulièrement recours aux magazines de programmes pour s'aider dans la sélection des émissions du jour ou de la semaine. À une époque où, le soir, « certaines grandes émissions de variétés, certaines dramatiques ${ }^{6}$ radiophoniques vidaient les cinémas ${ }^{7}$, l'intitulé de chaque émission contribue à créer un horizon d'attente: il la dote d'une forte dimension romanesque et donc divertissante ${ }^{8}$. D'un point de vue strictement thématique, les titres suffisent déjà à donner une indication sur le type d'histoire privilégié dans l'émission : ceux-ci renvoient le plus souvent aux grands personnages, grands événements, faits divers, énigmes de l'histoire et destins exceptionnels. Decaux, Castelot, Colin-Simard puis Chiappe s'inscrivent dans la filiation de G. Lenotre en prêtant une forte attention aux éléments anecdotiques et à la " petite histoire $»^{9}$.

5 La catégorisation des personnages évoqués nous permet de voir que près de $30 \%$ des émissions de l'échantillon convoquent des «souverains" (des monarques, des empereurs et leur entourage familial direct) ; plus de $23 \%$ font figurer des personnalités jouant un rôle politique, diplomatique ou judiciaire (présidents, ministres, conseillers, députés mais aussi dictateurs). Des militaires, chefs de guerre ou policiers apparaissent dans près de $20 \%$ des émissions et l'on compte aussi des journalistes, écrivains, intellectuels ou organisatrices de salons littéraires dans quasiment $15 \%$ des émissions. C'est donc une histoire par le haut que propose La Tribune de l'Histoire en racontant les événements historiques principalement du point de vue des élites ou bien de quelques 
destinées exceptionnelles (inventeurs, explorateurs, etc.). À ce titre, il est intéressant de souligner que l'on dénombre plus d'émissions centrées sur des figures "marginales» (6\% - criminels, prostituées, vagabonds ou encore bagnards) que d'évocations consacrées à des personnages «ordinaires", notamment paysans, travailleurs ou ouvriers ${ }^{10}$.

6 Il faut également noter que les thèmes abordés dans l'émission concernent majoritairement l'histoire contemporaine et française. $68 \%$ des émissions du corpus se rapportent à des événements historiques situés entre 1789 et le $\mathrm{xx}^{\mathrm{e}}$ siècle. Précisons toutefois que l'intérêt pour la période contemporaine est décroissant à mesure que l'on s'approche du temps présent : 150 émissions (soit 33\%) abordent la période 1789-1848, 107 (24\%) évoquent des événements compris entre 1848-1914 et 93 (21\%) vont au-delà de 1914. L'époque moderne n'est pas en reste puisqu'elle est mentionnée dans environ $30 \%$ des émissions, alors que les périodes médiévale et antique apparaissent dans moins de $5 \%$ du corpus.

7 Sur le plan géographique, 55\% des émissions sont exclusivement consacrées à la France et près de $30 \%$ abordent l'histoire d'un ou plusieurs pays européens (Russie incluse). Si des espaces extra-occidentaux sont traités en de rares occasions c'est souvent parce que le sujet a également une attache française : il est possible de parler du Maghreb ou $\mathrm{du}$ Moyen-Orient, mais pour évoquer Lyautey ou Lawrence d'Arabie, et si l'on mentionne l'Afrique, c'est au sujet de la mission Marchand à Fachoda ou de la mort du prince impérial en Afrique du Sud en 1879. Par ailleurs, ce déséquilibre dans les zones géographiques traitées est couplé à une vision parfois caricaturale des peuples noneuropéens, comme le montre le commentaire décrivant le peuple mongol dans une émission sur Gengis Khan diffusée en 1965: "Le Mongol est probablement l'être humain le plus sale du monde entier. [...] Il y a à cela une raison psychique, le Mongol est attaché à son animalité, or, l'animal ne se lave pas ». Dans cette évocation rédigée par Alain Decaux à partir de récits de voyages d'Occidentaux qui ne sont ni ethnologues ni historiens, l'accent mis sur l'altérité et l'animalité des Mongols, renforcée par l'usage du singulier - «le Mongol »-, rend le propos caricatural. Cela reflète, de la part des producteurs de l'émission, une conception très européo-centrée de l'histoire, que confirment des propos tenus par André Castelot en 1982: « en dehors de l'histoire française et de l'histoire anglaise qui sont passionnantes, vous avez l'histoire espagnole qui est intéressante également. Mais il $\mathrm{y}$ a tellement de pays où l'histoire n'est pas passionnante parce qu'il n'y a pas une continuité. $»^{11}$ Cette remarque témoigne plus d'une méconnaissance et d'un désintérêt pour ces aires culturelles que d'une réalité scientifique : en effet, les processus de décolonisation ont suscité un certain nombre de travaux historiques sur les sociétés non-européennes, renforcés par l'émergence de l'histoire globale et des postcolonial studies à partir des années 1970-1980. Mais l'approche événementielle et personnifiée d'une histoire essentiellement française que l'on retrouve dans la Tribune de l'histoire est aussi liée au format de l'émission : le choix de recourir à l'évocation scénarisée pour attirer un grand nombre d'auditeurs "surdétermine une écriture romanesque " ${ }^{12}$ et implique la mise en scène de personnages facilement identifiables par le public. 


\section{Séduire le public en s'assurant de la clarté du récit : possibilités et contraintes narratives de la radio}

8 Pour parvenir à fidéliser leur public, les producteurs de la Tribune de l'histoire ont dû se familiariser avec les contraintes de la radio tout en développant une écriture tenant compte des possibilités de ce média. Inspirés par les dramatiques qui connaissent un grand succès à la radio et guidés par les codes du théâtre radiophonique, Decaux, Castelot et Colin-Simard parviennent à se constituer un style propre en développant des "évocations scénarisées ». Si le format de l'émission ne change pas du milieu des années 1950 à l'arrêt de la Tribune en 1997, les producteurs ont, en revanche, constamment cherché à renouveler leurs procédés narratifs pour capter l'attention du public dès les premières secondes de l'évocation. Dans les dix émissions analysées, on retrouve le plus souvent un récit-cadre assuré par un dialogue entre deux protagonistes, selon des formules nombreuses: interrogatoire de procès, reportage dans le passé, entretien avec un personnage historique âgé qui raconte sa vie à un interlocuteur anonyme et intemporel, narration par un témoin ou un récitant externe à l'histoire. Ce récit-cadre, qui sert de trame narrative, est ensuite illustré par des scènes jouées par des comédiens et comédiennes.

9 Les textes sont rédigés de manière à encourager le public à vivre et ressentir les événements évoqués. Ainsi l'émission sur Gengis Khan commence-t-elle par une longue description de la steppe par un narrateur et se poursuit-elle par une présentation des us et coutumes du peuple mongol pour permettre l'immersion de l'auditeur dans l'histoire qui lui est proposée. Ce décor peut aussi être amplifié par des bruitages, bien que la palette de sons utilisée dans les émissions soit assez réduite: on remarque surtout des bruits de sabots, de portes, de pas ou de foule et une utilisation récurrente de la réverbération pour suggérer l'ambiance d'une église ou des débats à l'Assemblée. Les personnages eux-mêmes tentent parfois de communiquer une atmosphère ou leur ressenti, en recourant à des verbes de perception ou de sensation, comme Aliénor d'Aquitaine se souvenant de son mariage avec Louis VII, roi de France : " Je les entends encore ces acclamations qui nous ont salués à la sortie de la cathédrale » dit-elle sur un tapis sonore de cloches et de foule, « il faisait très chaud ce 25 juin 1137 ». Pour donner plus de force et de dynamisme à certains témoignages, l'auditeur peut aussi être interpellé directement par les personnages historiques. Dans l'émission sur les mutineries de 1917, un poilu originaire des Landes raconte sa guerre en prenant l'auditeur à témoin : "Vous pouvez pas vous figurer ce que c'était qu'la boue des tranchées [...] Et puis, on est parti pour Verdun. Pas besoin d'vous faire un dessin. Verdun, suffit d'prononcer l'nom. » Paul Crauchet, comédien originaire de Béziers, semble renforcer son accent du sud pour accentuer le parler « campagnard » du poilu : les « $\mathrm{r}$ » grasseyés et les syllabes tronquées cherchent à conférer une authenticité supplémentaire au récit.

10 Car, média sonore, la radio ne dispose que des voix et des sons pour évoquer la vie d'un personnage historique ou le déroulement d'un événement - elle doit donner à entendre des images. Pour cela, l'émission ne cherche pas à reproduire fidèlement le phrasé médiéval, la prononciation de l'époque baroque ou l'accent d'un paysan des Landes au début $\mathrm{du} \mathrm{xx}^{\mathrm{e}}$ siècle : il s'agit avant tout de suggérer. Les producteurs de l'émission se contentent de quelques formulations de circonstance pour signifier que le propos est ancré dans un contexte étranger ou passé comme lorsqu'Henri IV et Marie de Médicis 
s'adressent l'un à l'autre en s'appelant «ma mie » et « mon doux sire ». Car ce n'est pas la « ressemblance vocale » entre un acteur et le personnage qu'il incarne que l'équipe de l'émission cherche à atteindre mais plutôt une certaine "vraisemblance psychologique $\aleph^{13}$, quitte à basculer, parfois, dans le cliché : le comédien incarnant César Borgia a une voix puissante et virile pour coller avec l'image tyrannique donnée de lui ; à l'inverse, dans une autre émission, celui qui interprète Louis XIII a une voix timide pour renforcer l'idée qu'il a été écrasé par la régence de sa mère, tandis que la comédienne jouant Marie de Médicis emprunte du mieux qu'elle peut un fort accent italien.

11 Les réalisateurs successifs, Alain Barroux puis Georges Gravier, effectuent un important travail de mise en ondes afin de transformer le script des producteurs en véritable œuvre radiophonique. Car, si les tapuscrits ${ }^{14}$ comportent bien des indications "scéniques ", celles-ci sont plutôt laconiques : "musique ", " porte", " chevaux sur pavés", "trois coups de canon». Le réalisateur dispose alors d'une marge de manœuvre et de créativité pour apporter rythme et cohérence sonore à l'évocation, en mêlant voix, musiques et bruitages. Toutefois, dès l'étape de rédaction du script, les producteurs sont tenus de respecter un certain nombre de contraintes imposées par le média radio pour s'assurer de la clarté du récit. La première est celle de la durée l'évocation ne peut dépasser les quarante minutes qui lui sont allouées dans la grille de programmes, que le sujet abordé couvre une journée, une guerre, un règne ou un siècle entier. Pour cela, les producteurs ont régulièrement recours au flash-back initial : un personnage au crépuscule de sa vie - ou ressuscité pour l'occasion - revient sur les grandes étapes de son existence, mais dans l'ordre chronologique, afin de maintenir une certaine linéarité dans le récit, l'oreille accommodant moins bien que l'œil ${ }^{15}$. Les ellipses sont également un procédé courant, parfois accompagnées de virgules musicales pour rythmer l'évocation et créer des ambiances sonores. L'autre contrainte concerne les personnages: s'il n'est pas rare que les évocations comptent une quinzaine de rôles différents, chaque scène convoque rarement plus de trois à quatre protagonistes. Pour ne pas perdre l'auditeur, ils sont régulièrement nommés, ont une voix suffisamment caractéristique pour être reconnus d'une fois sur l'autre ou sont identifiables immédiatement par leur propos. Mais la nécessité de limiter le nombre de personnages conduit surtout à privilégier des scènes de dialogues, dans une pièce isolée, et donc à se concentrer sur la psychologie des figures historiques et sur les coulisses de l'histoire - en préférant Henri IV et Marie de Médicis dans l'intimité plutôt qu'Henri IV face à ses conseillers.

\section{Conférer une authenticité au propos : une émission en marge des évolutions de la discipline historique}

Dans les premières années, les producteurs répétaient couramment dans la presse spécialisée ne jamais prendre de libertés avec les faits historiques et ne rien écrire qui ne soit pas tiré des sources, présentant la radio comme un média leur permettant de rester au plus près de la vérité historique :

Ils comprirent que la radio pouvait atteindre une authenticité à laquelle ne saurait prétendre aucun art visuel. Elle épargne à l'auditeur une certaine gêne du truquage des yeux, qu'éprouve généralement le spectateur de cinéma. La radio se doit donc de profiter de cet atout et se refuser à la moindre tricherie. Les auteurs de la Tribune de l'histoire poussent l'honnêteté jusqu'à se défendre d'inventer la moindre réplique 
dramatique (les émissions d'été connaissent une rigueur moindre), et se réfèrent toujours à d'authentiques passages de lettres, à des citations, etc. L'histoire est à leurs yeux bien assez vivante pour se passer de reconstitutions fausses ou de coups de pouce dramatiques. Ils vont même jusqu'à prier les comédiens de «jouer " le moins possible. ${ }^{16}$ d'aller consulter les sources historiques pour y puiser leurs dialogues sans n'avoir rien à inventer, force est de constater qu'ils abandonnent cette exigence au fil du temps : l'impératif de produire chaque semaine une émission de radio originale les conduit à prendre quelques libertés, qu'ils ne reconnaissent qu'à demi-mot ${ }^{17}$. Lorsqu'ils écrivent leurs scripts, ils inventent des répliques, romancent certains événements et ont recours à ces «coups de pouce dramatiques » qu'ils dénonçaient initialement. Malgré cela, ils multiplient les procédés leur permettant d'assurer le public de l'authenticité et du sérieux de leurs émissions. Cela passe notamment par la mise en avant du contexte historique en confiant aux personnages le soin de se livrer eux-mêmes à des leçons d'histoire, au détour d'une réplique. Comme lorsque Henri Rochefort explicite le contexte politique dans lequel il commença sa carrière d'écrivain-journaliste tout en étant critique du régime de Napoléon III, dans les années 1850 : «La vérité est que l'Empire autoritaire avait muselé la presse. La censure se manifestait à tout instant : intolérante, omniprésente, tatillonne, souvent ridicule "; plus loin c'est son interlocuteur, anonyme et intemporel, qui décrit la seconde phase de l'Empire dans les années 1860-1870 : "Le carcan autoritaire se desserrait. L'empereur revenait aux opinions de sa jeunesse, à celles qu'il n'avait abandonné qu'à regret. L'Empire libéral se dessinait ».

Par ailleurs, en fidèles disciples de G. Lenotre, les producteurs de la Tribune accordent un grand soin à la mise en avant de la documentation historique : journaux intimes, lettres, mémoires, chroniques sont fréquemment convoqués et cités, aussi bien en début d'émission que dans le corps du scénario. Par exemple, dans l'émission sur Marie Bonaparte, arrière-petite-nièce de Napoléon Bonaparte et pionnière de la psychanalyse, des extraits de la correspondance entre le docteur René Laforgue et Freud au sujet de la princesse sont lus par un comédien. Il s'agit cependant d'éléments repris de l'ouvrage utilisé pour rédiger l'évocation ${ }^{18}$, car, la plupart du temps, les auteurs de La Tribune de l'Histoire ne font pas un travail de production des savoirs historiques. Les faits qu'ils exposent au public sont soumis à une double médiation : les producteurs s'appuient sur un ou plusieurs ouvrages qui présentent et interprètent les événements historiques une première fois, avant de procéder eux-mêmes à des sélections, des résumés ou des simplifications. Mais la mention régulière de documents d'archives précis, tout en renforçant l'impression de véracité de l'évocation, permet de faire oublier que les producteurs ne vont pas consulter des sources primaires pour chaque émission qu'ils préparent. authenticité supplémentaire aux événements évoqués. C'est notamment le cas dans l'émission "Les cent ans de Marianne ", préparée par Jean-François Chiappe, où sont convoqués Daniel Halévy, Jacques Bainville, Pierre Bécat et Jacques Chastenet pour raconter les débuts de la Troisième République. Seulement, il y a un certain paradoxe à ne mentionner que ces auteurs clairement situés à droite du spectre politique ${ }^{19}$ pour parler de l'installation du régime républicain, d'autant plus que leurs travaux datent majoritairement de la première moitié $\mathrm{du} \mathrm{xx}^{\mathrm{e}}$ siècle $^{20}$ et que d'autres publications plus

Belphégor, 19-1 | 2021 
récentes faites par des universitaires auraient pu être citées ${ }^{21}$. Nous remarquons par conséquent que les producteurs - et ici, Chiappe, qui ne cache pas sa proximité avec les courants monarchistes - opèrent des choix clairs dans les sujets traités, les lectures sur lesquelles ils s'appuient et les documents qu'ils mettent en avant.

Par ailleurs, alors que se développe l'histoire des femmes au cours de la seconde moitié $\mathrm{du} \mathrm{Xx}^{\mathrm{e}}$ siècle, les émissions centrées sur des femmes célèbres - reines ou princesses notamment - se limitent souvent à faire état de leurs malheurs et de leur vie amoureuse. En février 1983, l'émission sur Marie Bonaparte mentionne à peine son rôle dans le développement de la psychanalyse en France pour se focaliser sur sa frigidité et ses relations extra-conjugales. Par conséquent, alors que Decaux, Castelot, Colin-Simard puis Chiappe affirment s'appuyer sur des ouvrages récemment parus, leur émission reste assez largement déconnectée des renouvellements de la recherche historique, qu'il s'agisse d'histoire politique, religieuse, économique, sociale ou culturelle.

En cela, l'approche de l'histoire défendue par la Tribune ne fait pas l'unanimité : des critiques émanent, aussi bien au sein de la radio d'État que dans la communauté historienne. En 1960, le Comité des lettres et des œuvres dramatiques de la RTF invite Decaux, Castelot et Colin-Simard, « au lieu de traiter l'histoire sous l'angle des faits et des personnages, [à les] présent[er] plutôt selon les grandes perspectives économiques, sociales et culturelles ${ }^{22}$. Six ans plus tard, c'est Adrien Dansette qui émet un certain nombre de réserves face au Comité des programmes de l'ORTF. Ce membre de l'Académie des Sciences morales et politiques, qui effectue pourtant ses travaux historiques en marge du monde universitaire, estime que la Tribune emprunte trop à la petite histoire, traite ses sujets de manière trop "superficielle» et pas assez contextualisée, sans compter un certain nombre d'erreurs et d'inexactitudes : en s'appuyant sur l'écoute de plusieurs émissions, Dansette regrette de constater que la Tribune n'a « pour ainsi dire rien appris à l'auditeur sur les époques évoquées ${ }^{23}$.

C'est aussi cette conception de l'histoire que conteste Jacques Le Goff, universitaire très impliqué dans les renouvellements de la discipline puisqu'il est l'un des chefs de file de la Nouvelle Histoire. En tant que co-producteur des Lundis de l'histoire sur FranceCulture depuis 1968, il se soucie aussi de diffuser cette discipline dans la société et regrette l'abandon du grand public à la " pseudo-histoire des "énigmes de l'histoire" ou [...] des scandales de la vie privée des "grands" $»^{24}$, des approches qu'il juge peu profitables :

Je crois qu'il y a une volonté, et elle n'est pas innocente, de rabaisser l'Histoire, d'en faire une suite de petites histoires et de ragots qui maintiennent le public dans l'idée justement que ce qui fait l'histoire, ce sont des magouilles, de petites manigances. [...] De plus, cette Histoire, non seulement n'explique rien, mais elle maintient, je crois, très profondément et sur tous les plans, y compris sur les plans idéologiques et politiques, la sujétion du grand public, la sujétion de la masse, vis-àvis de forces qui peuvent le mieux les manipuler. Et donc à cet égard je crois qu'il faut dénoncer certaines façons de faire de l'Histoire. ${ }^{25}$

19 Ainsi, la pierre d'achoppement entre la communauté historienne, toute hétérogène soit-elle, et des figures médiatiques comme Decaux, Castelot et Chiappe réside dans leur manière de considérer l'histoire: dans la Tribune de l'histoire, l'événement passé n'est plus "un élément d'une réalité à interroger » mais une simple "mise à distance du présent $»^{26}$. La mise en scène a pour conséquence d'en «[reconduire] la charge mythologique " au lieu de la déconstruire comme a su le faire Duby avec son Dimanche de Bouvines ${ }^{27}$. Mais forts de leurs succès d'audience, les producteurs de la Tribune ne 
tiennent que peu compte des critiques émanant de la communauté historienne et préfèrent se concentrer sur leur public, qu'ils érigent en unique juge de la qualité de leurs émissions.

\section{Créer un lien avec les auditeurs}

20 La Tribune de l'histoire a rapidement su plaire à un large public: en 1959, le magazine Radio-Cinéma-Télévision titrait sur Decaux, Castelot et Colin-Simard, «ces trois hommes [qui] font aimer l'histoire à des millions d'auditeurs $»^{28}$ et les comparait à des scientifiques et à des détectives minutieux parvenant à rendre l'histoire attrayante. Plusieurs éléments peuvent nous aider à comprendre le succès de l'émission, reconduite sur quatre décennies sans changement de dispositif. Tout d'abord, il faut rappeler l'intérêt pour l'histoire qui touche la société française dans son ensemble au $\mathrm{xx}^{\mathrm{e}}$ siècle. L'enquête de René Kaës sur le rapport des ouvriers à la culture au tournant des années 1950-1960 montre que, pour cette catégorie de population, l'attrait pour l'histoire revêt plusieurs formes: un «goût [...] pour le «scandale» ou la petite histoire " ou encore une "curiosité pour une période favorable à l'évasion dans le temps et à la rêverie ", sans oublier "la recherche des progrès de l'homme à travers son histoire ${ }^{29}$. Ces thèmes et ces approches de l'histoire, que l'on retrouve régulièrement dans la Tribune, permettent au public d'apprécier le contenu de l'émission à plusieurs titres : à la fois pour son côté instructif (elle passe en revue de événements importants de l'histoire et l'attention portée aux détails ou aux petites anecdotes donne un sentiment d'érudition) et pour ses aspects divertissants (elle cultive la curiosité pour la vie des grandes figures du passé et les énigmes ou scandales de l'histoire ; l'écoute est rendue plaisante par la qualité radiophonique de sa mise en ondes). Auditrices et auditeurs ne manquent pas de signaler ce double caractère lorsqu'ils écrivent aux producteurs : " votre émission est la seule que nous écoutons ponctuellement avec un plaisir toujours renouvelé » explique un couple de Marseillais qui n'est pourtant pas "très amateurs de TSF "; "nous vous remercions de nous apporter chaque semaine une émission aussi intéressante et instructive » écrivent des habitants de Saint-Nazaire, de même que cet étudiant en sciences de Talence qui exprime ses « reconnaissantes félicitations » pour ce que La Tribune lui apporte « sur les deux plans de la détente et de l'instruction générale $»^{30}$.

21 Par ailleurs, pour établir une connivence avec le public, les producteurs donnent des clés de lecture de leurs propres débats en mettant régulièrement en scène leurs affiliations et divergences politiques. Ainsi Decaux confesse-t-il, dans l'émission sur la naissance de la Troisième République, « mon cœur est républicain, on le sait » de la même manière que Chiappe affirme sa foi dans l'émission sur Lucrèce Borgia. Bien informé du positionnement de chacun, le public peut obtenir une meilleure lecture des débats qui les opposent parfois en fin d'émission ${ }^{31}$. La mise en avant de sensibilités politiques variées devient aussi, selon les producteurs, un gage d'objectivité pour l'émission, Chiappe étant monarchiste, Castelot à droite et Decaux se réclamant de la " gauche Victor Hugo ".

22 Au-delà de la dimension politique, ils construisent aussi leur propre système de légitimation intellectuelle. Introductions et débats sont l'occasion pour eux de faire référence à des émissions sur des sujets connexes ou de citer un invité régulier de l'émission en louant sa qualité d'historien et son sérieux. Alors que leur réseau d'invités 
laisse de côté une large partie de la communauté historienne professionnelle, dont les noms ne sont jamais cités, ce jeu de références internes à l'émission donne ainsi l'impression qu'ils traitent tous les sujets et que les auteurs qu'ils convient sont bien représentatifs de la discipline historique dans sa variété de champs et de démarches. De plus, bien qu'aucun des trois producteurs n'ait reçu de formation d'historien, leur présence sur plusieurs supports médiatiques liés à la thématique historique suffit à leur décerner ce titre. En 1983, un sondage publié par L'Express sacrait Alain Decaux historien préféré des Français : à la question « Y a-t-il un ou plusieurs historiens, morts ou vivants, que vous aimez ou qui vous font aimer l'Histoire ? Lesquels? », Decaux était cité 251 fois, Castelot 73 fois, Victor Hugo 40 fois, Emmanuel Le Roy Ladurie 4 fois et Georges Duby comme Henri Amouroux 3 fois $^{32}$. Mais, dans un contexte de crise de l'enseignement de l'histoire, l'historien Pierre Nora préférait qualifier Decaux d'« instituteur national » pour mieux souligner son «rôle de substitution vis-à-vis d'une école qui ne joue plus son rôle " ${ }^{33}$. En effet, les sujets abordés dans l'émission renvoient à une culture historique scolaire héritée de la Troisième République, axée sur un roman national mêlant grandes figures historiques et batailles militaires ${ }^{34}$, à laquelle les producteurs ont ajouté des composantes plus divertissantes comme la petite histoire ou les énigmes de l'histoire.

Mais les années 1980 semblent ouvrir vers une conciliation entre l'histoire universitaire - qui, au demeurant, ne constitue pas une entité homogène - et le travail de figures médiatiques comme Decaux et Castelot. Cette forme de « conciliation » est permise par plusieurs facteurs. D'une part, les historiens professionnels commencent à se départir de leur méfiance à l'égard des médias audiovisuels et comprennent leur importance pour susciter une curiosité de l'histoire. Sans réellement approuver le type d'histoire mis en avant par la Tribune de l'Histoire, ils reconnaissent qu'il peut y avoir « diverses façons d'aller à l'Histoire $»^{35}$ et que de telles émissions " touchent une fibre dans le public » en s'emparant de toute une mythologie historique ${ }^{36}$ dont elles exploitent les ressorts dramatiques et romanesques. D'autre part, la querelle s'atténue aussi par le fait que les universitaires se font leur propre place dans le monde médiatique en créant des revues et des émissions de vulgarisation dans lesquelles ils assurent eux-mêmes la transmission des savoirs historiques issus des recherches les plus récentes ${ }^{37}$. Mais alors que de nouveaux lieux de diffusion de l'histoire émergent, la Tribune de l'histoire continue d'être reprogrammée d'année en année.

\section{La fin de la doyenne des émissions de radio}

En décembre 1996, une fête est organisée au Procope pour célébrer le $45^{\mathrm{e}}$ anniversaire de la Tribune de l'Histoire: la plus ancienne des émissions de la radio publique est célébrée avec champagne et bougies, en présence de ses trois producteurs, mais aussi de Michel Boyon, président de Radio France, et de Jacques Santamaria, directeur des programmes de France Inter. La presse généraliste se fait l'écho de l'événement, retraçant les débuts du programme et signalant l'exceptionnelle longévité dont il a su faire preuve $^{38}$. La fin de la Tribune n'est pas à l'ordre du jour et, en interne, des propositions sont même faites au trio pour moderniser l'émission et la rendre plus réactive à l'actualité. Cependant, l'émission s'arrête quelques mois plus tard, en 1997. $\mathrm{Au}$ cours de l'été, des ennuis de santé contraignent Alain Decaux à « renoncer à cette multiplication de tâches parallèles qui a toujours accompagné [sa] vie 
professionnelle $"^{39}$ : il met fin à sa participation à l'émission, une décision qui entraîne aussi le départ d'André Castelot, de quatorze ans son aîné. De son côté, Jean-François Chiappe, le plus jeune du trio, souhaite poursuivre son activité radiophonique, tout en convenant qu'il n'est pas possible de conserver le titre d'une émission largement incarnée par Decaux et Castelot. Il s'associe à Jean Favier et Arlette Lebigre, tous deux universitaires, pour créer Questions pour l'histoire, diffusée le samedi soir à 20 heures sur France Inter. Le programme, qui se poursuit jusqu'en 2006, s'inscrit dans la continuité de la Tribune, aussi bien dans les sujets traités que dans la forme de l'évocation suivie d'un débat.

La Tribune de l'histoire a su traverser les décennies sans connaître de modification réelle dans sa structure : du milieu des années 1950 jusqu'en 1997, c'est par la voix de comédiens et de comédiennes que sont mis en scène les épisodes du passé. Si les destins exceptionnels, les grands événements, les controverses et énigmes irrésolues de l'histoire permettent de répondre autant à un désir d'évasion qu'à une volonté d'érudition de la part du public, l'accent mis sur la dimension émotionnelle et fortement personnalisée d'une histoire principalement française et contemporaine, conduit les auteurs de l'émission à délaisser des pans entiers de la production historiographique récente. Et pourtant, aux yeux du « grand public », Decaux, Castelot, et, dans une moindre mesure, Colin-Simard et Chiappe, ont su incarner la figure de l'historien pendant plusieurs décennies, leurs voix et leurs visages restant durablement associés à une histoire plaisante, attractive, parfois énigmatique, mais dans l'ensemble rassurante. En effet, la succession de règnes et de batailles donne l'illusion de la continuité historique et les énigmes et scandales, dont l'issue n'a que peu d'impact sur le présent, viennent détourner la société de ses inquiétudes quant à l'avenir. En cela, dans un contexte de renouvellement de la discipline historique, de crise de son enseignement et d'interrogation sur ses rapports avec la mémoire, la Tribune de l'histoire a su symboliser la rassurante stabilité d'une certaine vision du passé.

\section{ANNEXES}

Liste des émissions écoutées :

- «La Fayette fut-il un grand homme: pour ou contre?», 28/10/1956, évocation écrite par Alain Decaux

- « Concino Concini », 05/07/1961, évocation écrite par André Castelot

- «Gengis Khan », 14/11/1965, évocation écrite par Alain Decaux

- « Henri de Rochefort », 17/12/1969, évocation écrite par Alain Decaux

- «Les cent ans de Marianne ", 18/01/1975, évocation écrite par Jean-François Chiappe

• « Un mutiné de 1917 », 03/02/1979, évocation écrite par Alain Decaux

- « Marie Bonaparte », 05/02/1983, évocation écrite par Alain Decaux

- «Aliénor d'Aquitaine », 23/04/1988, évocation écrite par Alain Decaux 
- «Tendre et dramatique Lucrèce Borgia », 08/05/1993, évocation écrite par André Castelot

• «L'enfance de Louis XIII », 14/06/1997, évocation écrite par André Castelot

\section{NOTES}

1. Entretien d'Alain Decaux avec Agathe Mella in Guy ROBERT (dir.), «La Tribune de l'Histoire [dossier] », Cahiers d'Histoire de la Radiodiffusion, mars 1995, nº 44, p. 80.

2. La station diffuse alors surtout des émissions de variétés, de la musique légère et des dramatiques. Pour un aperçu de la programmation de la station en 1953, voir Édith-Hélène BOUSSER-ECK, La Radiodiffusion française sous la IV République. Monopole et service public (août 1944décembre 1953), thèse de doctorat d'histoire, Paris X Nanterre, 1997, p. 643.

3. Pour cela, les données de dix saisons de l'émission ont été recensées - soit 445 émissions diffusées sur les années suivantes: 1956-1957, 1960-1961, 1965-1966, 1969-1970, 1974-1975, 1978-1979, 1983-1984, 1987-1988, 1992-1993, 1996-1997. À partir des titres de chaque émission, des notices de la base de données Hyperbase de l'Inathèque ou des courts synopsis publiés dans Radio Cinéma Télévision puis dans Télérama, nous avons pu déterminer les périodes historiques et zones géographiques traitées ou encore le profil des principaux personnages évoqués pour une majorité d'évocations. Lorsque ces éléments ne suffisaient pas, nous avons procédé à une écoute de l'émission.

4. Pour une analyse plus poussée, un échantillon de dix émissions - une pour chacune des dix saisons mentionnées précédemment (voir liste en annexes) - a été retenu en prêtant attention à plusieurs éléments :

- le dispositif de chaque évocation (dialogues, narration par un récitant, lecture d'extraits d'ouvrages, utilisation de musique ou de bruitages, etc.)

- la « mise en scène » de l'événement évoqué ou de la vie du personnage traité (récurrence de verbes d'action ou de perception; parler et vocabulaire des comédiens ; mise en avant de connaissances et de sources historiques ; importance accordée aux coulisses de l'histoire et notamment à la vie intime des personnages; valorisation d'une histoire divertissante, savante, politique, etc.)

- le débat qui suit les émissions (modalités de présentation de l'invité et de ses ouvrages; existence de désaccords entre les producteurs ou leur invité(e) ; mention des auditeurs ; vision de l'histoire mise en avant par les producteurs ; référence à d'autres émissions)

D'autres émissions ont également été écoutées pour vérifier les observations faites sur cet échantillon réduit.

5. Alain DECAUX, Le Tapis rouge, Paris, Perrin, 1992, p. 16.

6. Héritières du théâtre radiophonique, les dramatiques sont des pièces écrites ou adaptées spécifiquement pour la radio en exploitant les moyens d'expression propres à ce média. Elles peuvent aussi être déclinées en feuilletons.

7. Alain DECAUX et Pierre NORA, «Alain Decaux raconte... Alain Decaux », Le Débat, 1984, n 30, p. 45-80.

8. Matthieu Letourneux fait cette analyse à propos des articles du magazine Historia - dont Decaux, Castelot et Chiappe ont d'ailleurs été des contributeurs prolifiques - in Matthieu LETOURNEUX, «Quand l'Histoire courtise le roman: la revue Historia comme exemple d'Histoire romanesque ", Romanesque, 2008, $\mathrm{n}^{\circ}$ 4, p. 83-97.

9. Reposant sur l'utilisation d'anecdotes et de détails précis, la " petite histoire » est un mode de récit dans lequel « on accède toujours au passé par des personnages, par des biographies, et donc par des noms propres", Alain DELISSEN, «L'opération petit-historiographique. G. Lenotre, 
L'Impénétrable Secret du sourd-muet mort et vivant (1929), Vieilles maisons, vieux papiers (1906) et La Révolution française (2010) ", Écrire l'histoire, 2017, nº 17, p. 191.

10. Les résultats que nous obtenons recoupent ceux publiés par Guy Robert à partir des données de 394 émissions diffusées entre janvier 1979 et décembre 1986: Guy Robert, "Situations, acteurs: le spectacle sonore de l'histoire», in Guy ROBERT (dir.), «La Tribune de l'Histoire [dossier] », Cahiers d'Histoire de la Radiodiffusion, op. cit., p. 22-25. Comme il n'a pas toujours été possible d'attribuer une unique catégorie à chaque émission, la somme des pourcentages donnés peut dépasser $100 \%$. En effet, une même évocation scénarisée peut renvoyer à plusieurs profils de personnages, plusieurs zones géographiques ou plusieurs périodes historiques: l'émission « Napoléon et les Allemands» (15/10/1969) concerne aussi bien la France que l'Allemagne ; celle sur «Suez avant Lesseps » (28/06/1975) propose un aperçu transversal de l'histoire du canal, de l'antiquité à la période contemporaine; l'évocation «De Léopold II à Mobutu » (07/12/1996) convoque aussi bien des figures de souverains, que d'hommes politiques ou d'aventuriers.

11. Agathe MELLA, «Entretien. André Castelot: "Nous avons une histoire d'une richesse extraordinaire" », in Guy ROBERT (dir.), « La Tribune de l'Histoire [dossier] », Cahiers d'Histoire de la Radiodiffusion, op. cit., p. 78.

12. Anne-Marie THIESSE, Le roman du quotidien : lecteurs et lectures populaires à la Belle-Époque, Paris, Seuil, coll. « Points Histoire », n²77, 2000, p. 126.

13. Guy ROBERT, «Entretien. Georges Gravier: «L'imagination de l'auditeur est une composante de l'oeuvre radiophonique », Guy ROBERT (dir.), «La Tribune de l'Histoire [dossier]», Cahiers d'Histoire de la Radiodiffusion, op. cit., p. 111.

14. Le département des Arts du spectacle de la BnF conserve 500 tapuscrits de l'émission entre 1957 et 1982. L'IMEC conserve près de 600 scénarios allant de 1956 à 1997 dans le fonds Alain Decaux (467DCA).

15. GuY ROBERT, « Entretien. Georges Gravier : «L'imagination de l'auditeur est une composante de l'œuvre radiophonique » ", Guy ROBERT (dir.), «La Tribune de l'Histoire » [dossier], Cahiers d'Histoire de la Radiodiffusion, op.cit., p.118.

16. C.-M. TRÉMOIS, «Le micro peut-il être un bon historien ?", Radio Cinéma Télévision, n²95, 11 septembre 1955, p.6.

17. Agathe Mella, «Entretien. André Castelot: «Nous avons une histoire d'une richesse extraordinaire » ", Guy ROBERT (dir.), « La Tribune de l'Histoire [dossier] », Cahiers d'Histoire de la Radiodiffusion, op. cit., p. 77. Interrogé en 1982, André Castelot apporte quelques légères nuances au discours qu'ils tenaient auparavant sur le respect scrupuleux de la vérité historique (je souligne) : «Quand on l'écoute à la radio [...], on se dit: "Mon Dieu, ça s'est passé comme ça! Presque comme ça" (Soyons modeste, l'histoire est une approximation). Mais tout de même, c'est un plaisir double que de pouvoir se dire : «[...] ces paroles-là ont été prononcées autrefois ». Ce n'est pas de l'invention, ce n'est pas de l'imagination, c'est vraiment le reflet de la vérité. ».

18. Célia BERTIN, La Dernière Bonaparte, Paris, Librairie Académique Perrin, 1982, p. 251-256. Pour reproduire ces extraits, l'autrice s'est elle-même appuyée sur: André BOURGUIGNON, «Correspondance Sigmund Freud - René Laforgue, Mémorial d'une rencontre », Nouvelle Revue de Psychanalyse, n¹5, printemps 1977, p. 235-374.

19. Pierre Bécat est un avocat et écrivain monarchiste, ancien membre de l'Action française, mort en 1993 ; Daniel Halévy est issu de l'orléanisme, proche de Barrès et Maurras et ses ouvrages sont "sympathiques au régime de Vichy " selon Olivier Lévy-Dumoulin ; Jacques Bainville, chroniqueur de L'Action française, appartient à la droite monarchiste. Par ailleurs, Bainville, Chastenet et Halévy sont rattachés à l'Institut de France (Académie française et/ou Académie des sciences morales et politiques), où Olivier Lévy-Dumoulin a souligné la forte présence des orléanistes puis des proches de l'Action française. Se reporter à Olivier DUMoulin, « Histoire et 
historiens de droite ", in Jean-François SIRINELLI (dir.), Histoire des droites en France. 2. Cultures, [1ère éd. : 1992]., Paris, Gallimard, coll. « Tel », 2006, p. 327-398.

20. Jacques Bainville, La Troisième République (1935) ; Daniel Halévy, Pour l'étude de la Troisième République (1937) ; Jacques Chastenet, Histoire de la Troisième République, (7 vol.: 1952-1963) ; l'ouvrage de Pierre Bécat n'est pas cité.

21. Pour n'en citer que deux: Jean-Pierre AzÉmA, Michel WINock, La Troisième République, Paris, Calmann-Lévy, 1970 ; René RÉMOND, La Vie politique en France depuis 1789. Tome $2: 1848-1879$, Paris, Armand Colin, 1969.

22. Comité des lettres et des œuvres dramatiques, procès-verbal de la séance tenue le 8 juin 1960, AN 19900214/15.

23. Comité des programmes de l'ORTF, procès-verbal de la séance tenue le 20 janvier 1966, AN $19900214 / 43$.

24. "Une science en marche, une science dans l'enfance » in Jacques LE GOFF (dir.), La Nouvelle histoire, Paris, C.E.P.L., coll. « Les Encyclopédies du savoir moderne », 1978, p. 13.

25. Guy ROBERT, « Entretien avec Jacques Le Goff : L'histoire centrée sur les grands hommes peut favoriser la sujétion des masses », [Dossier : « Ils racontent l'histoire. Gens de radio, historiens : 5 grands conteurs »], Multiplex : Radio France International, 1984, p. 129.

26. Matthieu LETOURNEUX, «Quand l'Histoire courtise le roman; La revue Historia comme exemple d'Histoire romanesque », art. cit., p. 95.

27. Alain DECAUX et Pierre NORA, «Alain Decaux raconte... Alain Decaux », art. cit.

28. Lucien MALSON, "Ces trois hommes font aimer l'histoire à des millions d'auditeurs ", Radio Cinéma Télévision, n502, 30 août 1959, p. 11.

29. René KAËs, Les Ouvriers français et la culture, enquête 1958-1961, Paris, Dalloz, 1962, p. 204.

30. «Lettres reçues à la suite de l'émission sur le massacre de Katyn », 738 DCA 77/3, Fonds Alain Decaux, IMEC. En dehors de quelques exceptions, les courriers conservés concernent principalement l'émission sur le massacre de Katyn diffusée en trois volets en octobre-novembre 1966. Alors que les producteurs mentionnent fréquemment la grande quantité de lettres reçue chaque semaine pour cette émission, elles ne semblent pas avoir été conservées systématiquement, ni par les producteurs, ni par la chaîne qui diffusait l'émission.

31. Par exemple, le débat qui suit l'émission du 3 février 1979 sur les mutineries de 1917 est l'occasion de confronter les points de vue de Chiappe et Castelot, hommes de droite, face à l'invité Pierre Durand, journaliste communiste. C'est sur sa biographie du poilu Vincent Moulia, condamné à mort à tort pour une mutinerie, qu'Alain Decaux s'est appuyé pour rédiger l'évocation du jour. Mais Jean-François Chiappe récuse la référence à Pétain dans le sous-titre choisi par Durand « Vincent Moulia, les pelotons du général Pétain » : il rappelle que Pétain est « justement l'homme qui restaurera la confiance ", ce qu'appuie André Castelot, tout en étant sensible à la « confusion » que cela pourrait entraîner pour le public. Decaux, plus à gauche que ses deux collègues, ne prend pas part à cette discussion.

32. «Les Français jugent leur histoire ", L'Express, 19 août 1983. Les éléments de l'enquête ont été mis en ligne sur le site de l'hebdomadaire et sont consultables à l'adresse suivante : https:// www.lexpress.fr/culture/livre/1983-enquete-les-francais-jugent-leur-histoire_2094344.html [consulté le lundi 28 octobre 2019].

33. Alain DECAUX et Pierre NORA, « Alain Decaux raconte... Alain Decaux », art. cit.

34. En 1979, Alain Decaux était le grand défenseur du maintien d'une histoire traditionnelle (grandes figures et événements) et du retour de la chronologie dans les programmes scolaires, longtemps rejetés par les historiens et historiennes dans la lignée de l'école des Annales. Sur cette polémique et plus généralement sur l'enseignement de l'histoire en France, voir Patrick GARCIA et Jean LEDUC, L'enseignement de l'histoire en France: de l'Ancien régime à nos jours, Paris, Armand Colin, coll. « U Histoire », 2003, 319 p ; Laurence de DE COCK, Sur l'enseignement de l'histoire: 
débats, programmes et pratiques du XIXe siècle à aujourd'hui, Paris, Libertalia, coll. «Ceux d'en bas ", 2018, 329 p.

35. Guy ROBERT, « Entretien avec Jacques Le Goff : L'histoire centrée sur les grands hommes peut favoriser la sujétion des masses », art. cit.

36. Alain DECAUX et Pierre NORA, «Alain Decaux raconte... Alain Decaux », art. cit.

37. Entre 1968 et les années 1990, plusieurs universitaires ont rejoint l'équipe de production des Lundis de l'histoire: Jacques Le Goff, Denis Richet, Roger Chartier, Philippe Levillain, Michelle Perrot et Arlette Farge. Mais on peut aussi mentionner le lancement du magazine L'Histoire en 1978, dirigé par Michel Winock, ou encore l'adaptation télévisuelle du Temps des cathédrales de Georges Duby en 1980, quatre ans après celle de La Méditerranée de Fernand Braudel.

38. Colette Bolllon, "Anniversaire. André Castelot et Alain Decaux... à eux deux, 90 ans de "Tribune". La plus ancienne émission de radio fêtée dans le plus vieux café de Paris : c'est "La tribune de l'Histoire" qui célèbre son $45^{\mathrm{e}}$ anniversaire radiophonique ", La Croix, 7 décembre 1996, p. 21 ; Jean-Philippe CATINCHI, « La doyenne de la radio », Le Monde, 21 octobre 1996, p. 30.

39. Alain DECAUX, Lettre à Jacques Santamaria [dactylographiée], 31 juillet 1997 - IMEC, Fonds Alain Decaux, 738 DCA 774.

\section{RÉSUMÉS}

Diffusée de 1951 à 1997 sur les ondes de France Inter, La Tribune de l'Histoire a marqué le paysage radiophonique français par sa longévité et sa popularité. D’abord pensée comme un magazine faisant état de l'actualité de la discipline historique, l'émission s'est rapidement transformée en «évocation scénarisée » hebdomadaire évoquant l'affaire Rosenberg, les histoires d'amour du Vert Galant ou encore la chute du dernier tsar de Russie. Comprendre comment les producteurs de l'émission se sont appropriés le support radiophonique pour transmettre leur passion de l'histoire à des millions d'auditrices et d'auditeurs revient d'abord à analyser le type d'histoire mis en avant dans $L a$ Tribune et les techniques développées pour la mettre en scène. Si l'émission connaît un grand succès, sa popularité n'empêche pas les critiques, notamment de la part d'historiens universitaires : en privilégiant la dramatisation de grands événements, d'énigmes ou de faits divers, l'émission reste en marge des avancées historiographiques de la seconde moitié du $\mathrm{xx}^{\mathrm{e}}$ siècle. En cela, elle constitue un intéressant point d'entrée dans les débats et conflits qui ont pu opposer, à cette période, les universitaires aux vulgarisateurs

\section{INDEX}

Mots-clés : histoire, vulgarisation, récit, radio, populaire, légitimation, université, savoir 